\title{
RUNX3 plays an important role in mediating the BMP9-induced osteogenic differentiation of mesenchymal stem cells
}

\author{
YUFENG WANG, QIAOLING FENG, CAIXIA JI, XIAOHUA LIU, LI LI and JINYONG LUO \\ Key Laboratory of Diagnostic Medicine Designated by The Chinese Ministry of Education, \\ Chongqing Medical University, Chongqing 400016, P.R. China
}

Received February 20, 2016; Accepted September 8, 2017

DOI: 10.3892/ijmm.2017.3155

\begin{abstract}
Although bone morphogenetic protein 9 (BMP9) is highly capable of promoting the osteogenic differentiation of mesenchymal stem cells (MSCs) both in vitro and in vivo, the molecular mechanisms involved remain to be fully elucidated. Runt-related transcription factor (RUNX)3 is an essential regulator of osteoblast/chondrocyte maturation. However, the exact role of RUNX3 in BMP9 osteoinductive activity is unknown. In this study, we sought to investigate the functional role of RUNX3 in the BMP9-induced osteogenic differentiation of MSCs. We found that BMP9 upregulated the endogenous expression of RUNX3 in MSCs. The overexpression or/and knockdown of RUNX3 both increased the levels of alkaline phosphatase (ALP) a marker of BMP9-induced early osteogenic differentiation. Nevertheless, matrix mineralization, a marker of BMP9-induced late osteogenic differentiation was enhanced by the overexpression of RUNX3, whereas it was inhibited by the knockdown of RUNX3. The BMP9-induced expression of osteogenic pivotal transcription factors [inhibitor of differentiation (Id)3, distal-less homeobox 5 (DLX5) and RUNX2)] was further increased by the overexpression of RUNX3; however, it was reduced by the knockdown of RUNX3. However, the expression levels of Id1 and Id 2 were both enhanced by the overexpression or/and knockdown of RUNX3. The BMP9-induced phosphorylation of Smad1/5/8 was increased with the overexpression of RUNX3, and yet was decreased with the knockdown of RUNX3. Collectively, our findings suggest that RUNX3 is an essential modulator of the BMP9-induced osteoblast lineage differentiation of MSCs.
\end{abstract}

Correspondence to: Dr Jinyong Luo, Key Laboratory of Diagnostic Medicine Designated by The Chinese Ministry of Education, Chongqing Medical University, 1 Yixueyuan Road, Chongqing 400016, P.R. China

E-mail: luojinyong888@hotmail.com

Key words: mesenchymal stem cells, bone morphogenetic protein 9, runt-related transcription factor 3 , osteogenesis

\section{Introduction}

Mesenchymal stem cells (MSCs) are multipotent cells capable of differentiating into myogenic, osteoblastic, chondrogenic or adipogenic lineages. MSCs can be isolated from a number of tissues, such as trabecular bone, the periosteum, synovium, adipose tissue, skeletal muscle and deciduous teeth, as well as from placenta and umbilical cord blood (1-7). Due to their wide availability and the characteristic of multipotent differentiation, MSCs have been widely used in bone tissue engineering in the clinical setting $(2-5,8)$.

Bone morphogenetic proteins (BMPs), which belong to the transforming growth factor- $\beta$ superfamily, are important in stem cell biology and regulate cell proliferation and differentiation during development $(9,10)$. Additionally, the roles of BMPs in bone and skeletal development are well recognized $(6,11)$. More than 20 BMPs have been identified, and BMP2, BMP4, BMP6 and BMP7 are of more osteoinductive ability (11). Recombinant human BMP2 and BMP7 proteins have been used in the clinical setting (12-15). However, it remains unclear as to whether BMP2 and BMP7 are the most potent BMPs in inducing osteogenic differentiation and bone formation. It has previously been demonstrated that BMP9 is more potent in promoting the osteogenic differentiation of MSCs both in vitro and in vivo (16). Many pivotal transcription factors, such as inhibitor of differentiation (Id)1, Id2, Id3, distal-less homeobox 5 (DLX5) and runt-related transcription factor (RUNX)2, are pivotal mediators of the BMP9-induced differentiation of MSCs (16). A variety of signaling pathways, such as the canonical BMP/Smad signaling pathway and non-canonical mitogen-activated protein kinase (MAPKs), have been identified to meditate BMP9-induced osteogenic signaling (17-19). We have also previously found that BMP type I receptors, ALK1 and ALK2, are involved in BMP9induced osteogenesis (20). Additionally, microRNAs (miRNAs or miRs) are important regulators of BMP9-induced osteogenesis (21-23). Despite these meaningful findings, BMP9 remains the least studied BMPs and the molecular mechanisms involved in BMP9 osteoinductive activity remain to be fully elucidated.

The RUNX proteins, which include RUNX1, RUNX2 and RUNX3, play an important role in cell growth and differentiation during embryonic development (24). The RUNX1 null phenotype leads to the early embryonic lethality of homozygous mice, demonstrating that RUNX1 is required for definitive 
hematopoiesis (25). RUNX2 acts as a key regulator during osteogenesis $(26,27)$. RUNX2 knockout leads to the impaired hepertrophy of chondrocytes followed by the complete absence of mineralized bone $(26,27)$. Previous studies on RUNX3 have mainly focused on the function of gastric tumor suppressor and central nervous system development $(28,29)$. RUNX3 also plays an important role in chondrocyte differentiation and bone formation (30-32). The expression of RUNX3 increases at the stage of prehypertrophic chondrocytes and is maintained in hypertrophic chondrocytes (32). However, it decreases in terminal hypertrophic chondrocytes, and the expression pattern of RUNX3 overlaps with RUNX2 (32). RUNX genes and proteins share structural and organizational features and all RUNX proteins are co-expressed in many skeletal elements (33-35). We have validated in our previous studies that RUNX2 is involved in the BMP9-induced osteogenic differentiation of MSCs $(16,36)$. In the present study, we examined the effect of RUNX3 on the BMP9-induced osteogenic differentiation of MSCs. Our results demonstrated that RUNX3 may play regulatory roles in the BMP9-induced osteogenic differentiation of MSCs by affecting the Smad signaling cascade.

\section{Materials and methods}

Cell lines, cell culture and chemicals. 293 (for amplification of adenoviruses), HCT116, C3H10T1/2, C2C12 cells were obtained from ATCC (Manassas, VA, USA) and maintained in complete Dulbecco's modified Eagle's medium (DMEM) supplemented with $10 \%$ fetal bovine serum and $100 \mathrm{U} / \mathrm{ml}$ streptomycin/penicillin at $37^{\circ} \mathrm{C}$ in a humidified atmosphere of $5 \% \mathrm{CO}_{2}$. Unless otherwise indicated, all chemicals were purchased from Sigma-Aldrich (St. Louis, MO, USA).

Construction of recombinant adenovirus. Recombinant adenoviruses expressing exogenous BMP9 (Ad-BMP9) and RUNX3 (Ad-RUNX3), and recombinant adenovirus expressing small interfering RNA (siRNA) targeting RUNX3 (Ad-siRUNX3) was generated using hte AdEasy system as previously described (37). Adenoviruses only expressing GFP (Ad-GFP) and RFP (Ad-RFP) were used as controls. Ad-RUNX3 or Ad-BMP9 were used to infect the MSCs with a multiplicity of infection (MOI) of 5. An MOI of 5 indicates 5 active viral particles per cell. To obtain the indicated MOI, we first counted the quantity of the active viral particles, then counted the cell number and finally calculated the volume of viruses according to the indicated MOI.

Preparation of BMP9 conditioned medium. BMP9 conditioned medium (BMP9-CM) was prepared as follows: briefly, HCT116 cells were seeded in $100 \mathrm{~mm}$ dish and infected with an optimal titer of Ad-BMP9. The culture medium was changed into serum-free DMEM at $8 \mathrm{~h}$ following infection. BMP9 conditioned medium (BMP9-CM) was harvested at 24 and $48 \mathrm{~h}$ after infection and used immediately.

Total RNA isolation, semi-quantitative PCR and reverse transcription-quantitative PCR $(R T-q P C R)$. Total RNA was isolated from the cells using TRIzol reagent (Invitrogen, Carlsbad, CA, USA) and used to generate cDNA hexamer (Takara Bio Inc., Otsu, Japan) and MMLV transcription reverse transcriptase (Promega, Sunnyvale, CA, USA). RT-PCR was carried out as previously described (17-19) and PCR primers (Table I) were designed using the Primer3 program. A touchdown cycling program for RT-PCR was carried out as follows: $94^{\circ} \mathrm{C}$ for $5 \mathrm{~min}$ for 1 cycle, $94^{\circ} \mathrm{C}$ for $30 \mathrm{sec}, 68^{\circ} \mathrm{C}$ for $30 \mathrm{sec}$, and $72^{\circ} \mathrm{C}$ for 12 cycles with decrease in $1^{\circ} \mathrm{C} /$ cycle and then at $94^{\circ} \mathrm{C}$ for $30 \mathrm{sec}, 55^{\circ} \mathrm{C}$ for $30 \mathrm{sec}$, and $72^{\circ} \mathrm{C}$ for $30 \mathrm{sec}$ for 18-27 cycles depending on the abundance of a given gene. qPCR based on SYBR Premix Ex Taq (Takara Bio Inc.) was carried out to amplify the interesting genes with Bio-Rad iQ5 instrument (Bio-Rad, Hercules, CA, USA). Gene expression results were analyzed using the $\Delta \Delta \mathrm{Ct}$ method. A RT-qPCR cycling program was carried out as follows: $95^{\circ} \mathrm{C}$ for $3 \mathrm{~min}$, $95^{\circ} \mathrm{C}$ for $3 \mathrm{sec}, 55^{\circ} \mathrm{C}$ for $30 \mathrm{sec}\left(36\right.$ cycles), $95^{\circ} \mathrm{C}$ for $10 \mathrm{sec}$, $65^{\circ} \mathrm{C}$ for $10 \mathrm{sec}$ and $95^{\circ} \mathrm{C}$ for $50 \mathrm{sec}$. Triplicate reactions were carried out for each sample.

Western blot analysis. For western blot analysis, the cells were lysed with RIPA buffer and cleared total lysate was denatured by boiling and loaded onto an $8-15 \%$ gradient sodium dodecyl sulfate-polyacrylamide gel electrophoresis (SDS-PAGE) gel. Following electrophoretic separation, the proteins were transferred onto an Immobilon-P membrane and blocked by super-block blocking buffer and probed by primary antibodies, followed by incubation with secondary antibody. The proteins of interest were detected using a SuperSignal West Pico Chemiluminescent Substrate kit. The following primary antibodies were obtained from Santa Cruz Biotechnology, Inc. (Santa Cruz, CA, USA) as follows, anti- $\beta$-actin (sc-47778), anti-RUNX3 (sc-30197), anti-RUNX2 (sc-12488), antiDLX5 (sc-18151) and anti-Smad1/5/8 (sc-6031). The following primary antibodies were purchased from Cell Signaling Technology (Danvers, MA, USA) as follows, anti-phospho-p38 (\#4511), anti-p38 (\#9212), anti-phospho-ERK1/2 (\#4370), anti-ERK1/2 (\#4695). The dilution for all primary antibodies was 1:1,000. The following secondary antibodies were purchased from Zhongshan Golden Bridge (Guangzhou, China) as follows, peroxidase-conjugated rabbit anti-goat IgG (ZB-2306), peroxidase-conjugated goat anti-mouse $\operatorname{IgG}(\mathrm{ZB}-2305)$ and peroxidase-conjugated goat anti-rabbit IgG (ZB-2301). The dilution for all secondary antibodies was 1:5,000.

Measurement of alkaline phosphatase (ALP). ALP activity was assessed by a modified Great Escape SEAPChemiluminescence assay (BD Clontech, Mountain View, CA, USA) as previously described (17-19). For the chemilluminescence assays, each assay condition was performed in triplicate, and the results were repeated in at least 3 independent experiments. ALP activity was normalized to the total cellular protein level.

Measurement of matrix mineralization. Mineralized matrix nodules were stained for calcium precipitation by means of Alizarin Red S staining, as previously described (19). The cells were fixed with $0.05 \%(\mathrm{v} / \mathrm{v})$ glutaraldehyde at room temperature for $10 \mathrm{~min}$. After being washed with distilled water, the fixed cells were incubated with $0.4 \%$ Alizarin Red S for $5 \mathrm{~min}$, followed by extensive washing with distilled water. The staining of calcium mineral deposits was recorded under bright field microscopy. The results were repeated in at least 3 independent experiments. 
Table I. Sequences of primers for RT-PCR and RT-qPCR.

\begin{tabular}{lll}
\hline Gene name & \multicolumn{1}{c}{ Forward primer } & Reverse primer \\
\hline GAPDH & GGCTGCCCAGAACATCAT & CGGACACATTGGGGGTAG \\
Id & ACGACATGAACGGCTGCT & CAGCTGCAGGTCCCTGAT \\
Id2 & CAGCATCCCCCAGAACAA & TCTGGTGATGCAGGCTGA \\
Id3 & CTACGAGGCGGTGTGCTG & GCGCGAGTAGCAGTGGTT \\
DLX5 & CTCAGCCACCACCCTCAT & TGGCAGGTGGGAATTGAT \\
RUNX2 & GGTGAAACTCTTGCCTCGTC & AGTCCCAACTTCCTGTGCT \\
RUNX3 & CACCGGCAGAAGATAGAAG & GTCTGAGGAGCCTTGGATTG
\end{tabular}

RT-qPCR, reverse transcription-quantitative polymerase chain reaction; GAPDH, glyceraldehyde 3-phosphate dehydrogenase; Id1, inhibitor of differentiation 1; Id2, inhibitor of differentiation 2; DLX5, distal-less homeobox 5; RUNX3, runt-related transcription factor 3.

A

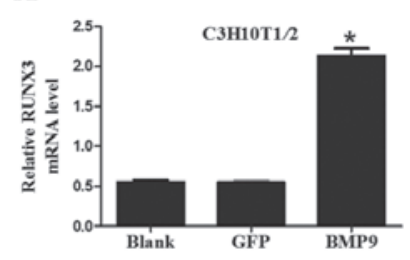

$\mathbf{E}$

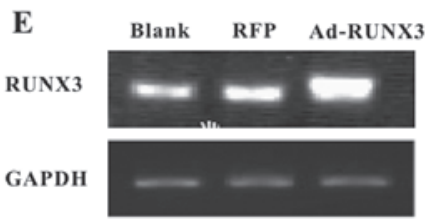

B
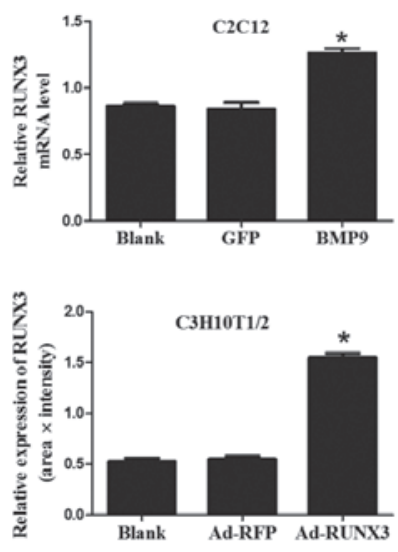

C
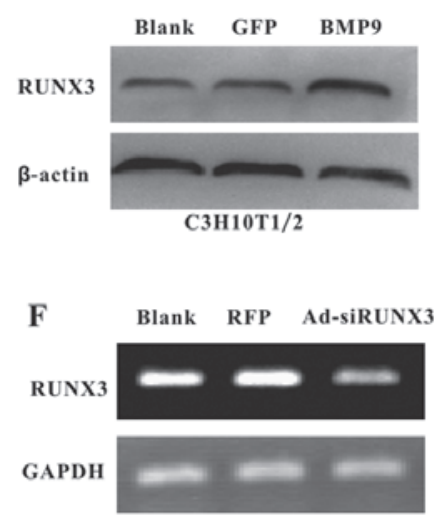

D
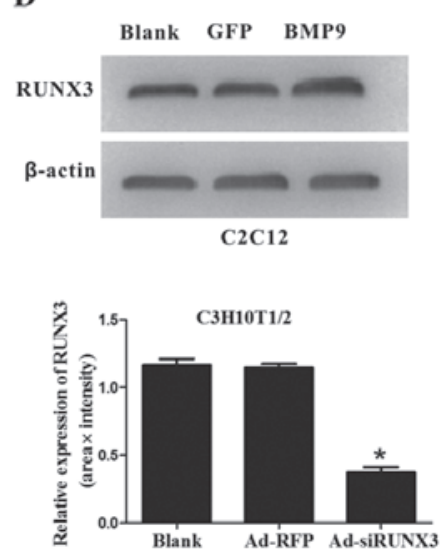

Figure 1. Bone morphogenetic protein 9 (BMP9) increaseS the endogenous expression of runt-related transcription factor 3 (RUNX3). C3H10T1/2 and C2C12 CELLS were infected with adenovirus (Ad)-GFP or Ad-BMP9. (A) BMP9 increaseD RUNX3 mRNA expression in C3H10T1/2 cells, as detected by RT-qPCR. (B) BMP9 increaseD RUNX3 mRNA expression in C2C12 cells, as detected by RT-qPCR. (C) BMP9 increased RUNX3 protein expression in C3H10T1/2 cells, as detected by western blot analysis. (D) BMP9 increased RUNX3 protein expression in C3H10T1/2 cells, detected by western blot analysis. C3H10T11/2 cells were infected with Ad-RFP, Ad-RUNX3 or adenovirus expressing small interfering RNA (siRNA) targeted against RUNX3 (Ad-siRUNX3). (E) Ad-RUNX3 increased RUNX3 mRNA expression in C3H10T1/2 cells, as detected by RT-qPCR. (F) Ad-siRUNX3 decreased RUNX3 mRNA expression in C3H10T1/2 cells as detected by RT-qPCR. " P $<0.05$ vs. blank and RFP.

Statistical analysis. Data were analyzed using One-way analysis of variance (ANOVA) followed by the Tukey's test. Data are presented as the means \pm SEM and statistical significance was indicated by $\mathrm{p}$-value $<0.05$.

\section{Results}

BMP9 upregulates the expression of endogenous RUNX3 in MSCs. First of all, we sought to determine whether BMP9 can affect the expression of endogenous RUNX3. We found that BMP9 effectively increased the mRNA and protein expression level of RUNX3 in C3H10T1/2 cells (Fig. 1A and C) and C2C12 cells (Fig. 1B and D). Subsequently, we successfully constructed recombinant adenovirus expressing RUNX3 (Ad-RUNX3) (Fig. 1E) and adenovirus expressing siRNA against RUNX3 (Ad-siRUNX3) (Fig. 1F). Our results indicate that BMP9 increases the expression of RUNX3 in MSCs.

Effect of RUNX3 on the BMP9-induced increase in the level of the early osteogenic marker, ALP. BMP9 increased RUNX3 expression in MSCs, and this prompted us to evaluate the effect of RUNX3 on the BMP9-induced osteogenic differentiation of MSCs. We infected the C3H10T1/2 and C2C12 cells with Ad-RFP or Ad-RUNX3, followed by treatment with BMP9$\mathrm{CM}$. The results revealed that the BMP9-induced ALP activity was further increased following transfection of the C3H10T1/2 cells (Fig. 2A and B) and $\mathrm{C} 2 \mathrm{C} 12$ cells (Fig. 2E and F) with Ad-RUNX3. To complement these results, we also infected the $\mathrm{C} 3 \mathrm{H} 10 \mathrm{~T} 1 / 2$ and $\mathrm{C} 2 \mathrm{C} 12$ cells with siRNA against RUNX3 (Ad-siRUNX3). Still, an increased level of ALP activity was observed in the $\mathrm{C} 3 \mathrm{H} 10 \mathrm{~T} 1 / 2$ cells (Fig. 2C and D) and $\mathrm{C} 2 \mathrm{C} 12$ cells (Fig. 2G and $\mathrm{H}$ ). Collectively, both the overexpression and/or knockdown of RUNX3 enhanced the BMP9-induced early osteogenenic differentiation of MSCs.

Effect of RUNX3 on the BMP9-induced increase of the late osteogenic marker, matrix mineralization. Although ALP is an early osteogenic marker, it is hardly an accurate late osteogenic marker. Thus, we sought to examine the effect of RUNX3 on the BMP9-induced late osteogenic marker, matrix 
A

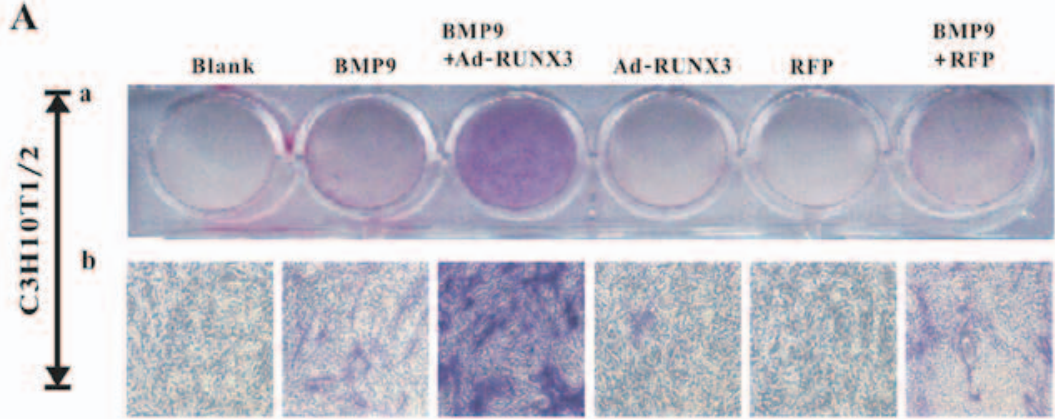

C

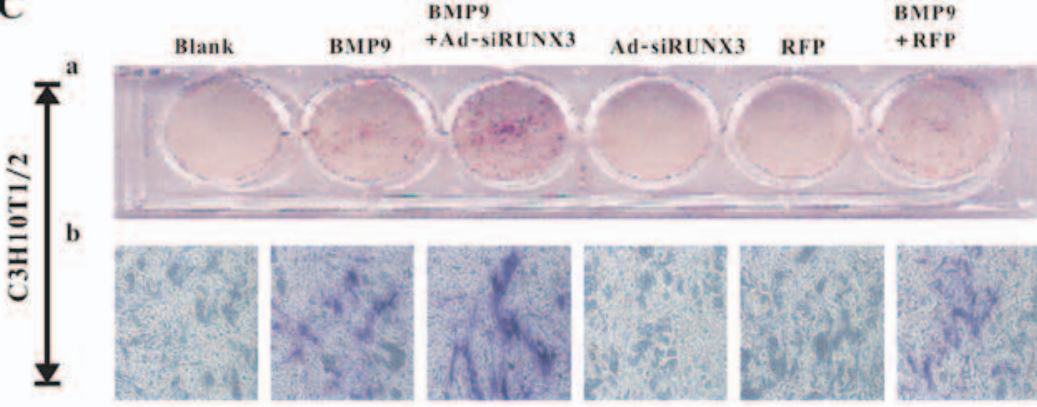

E

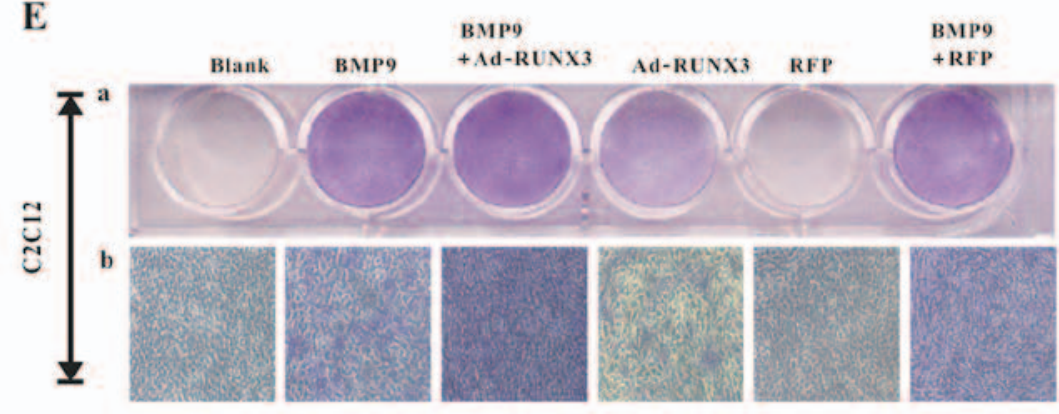

G

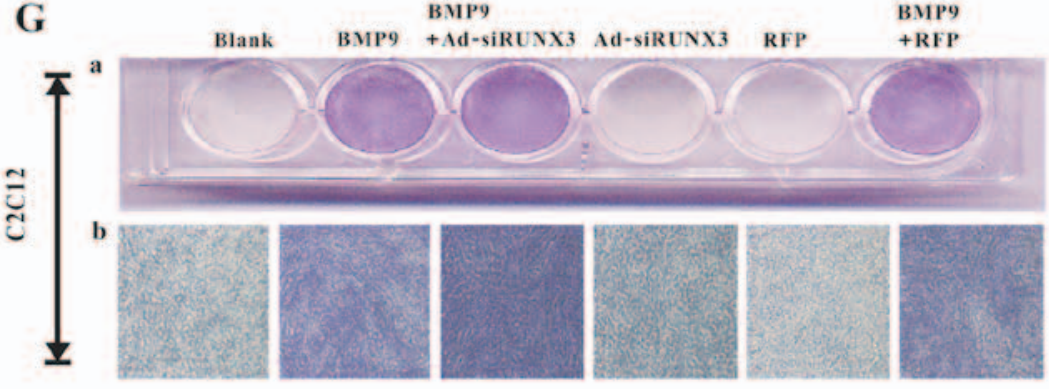

B

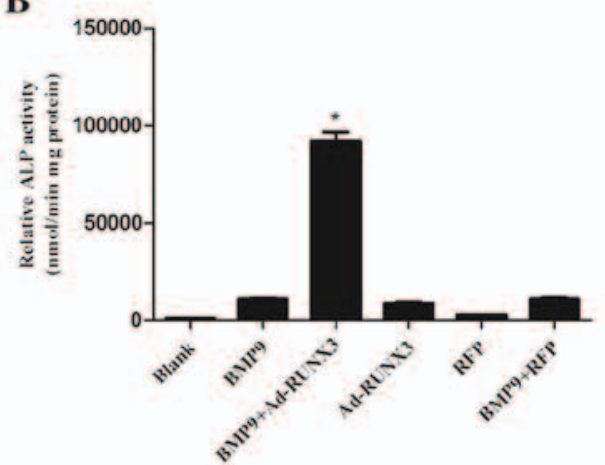

D

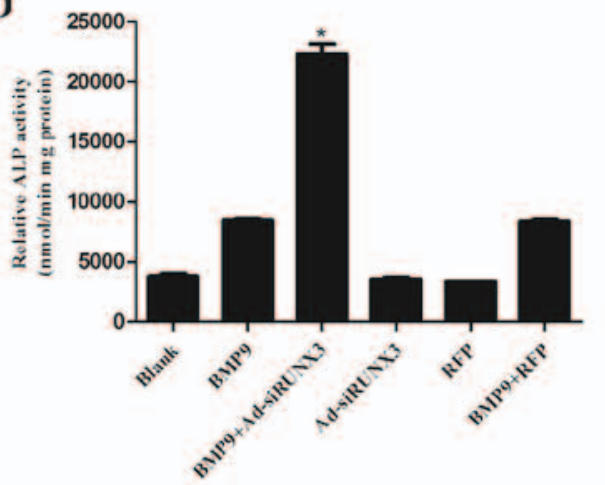

$\mathbf{F}$

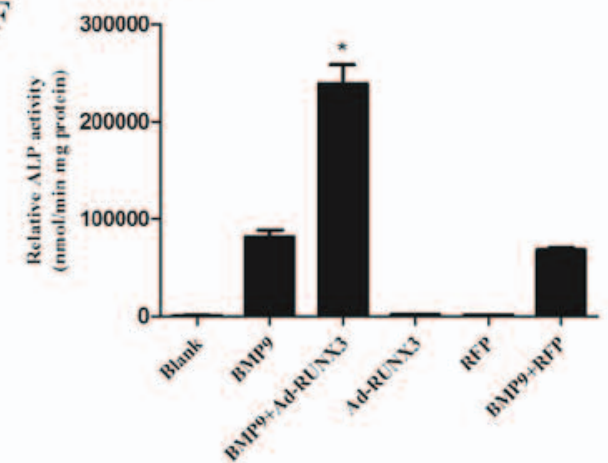

$\mathbf{H}$

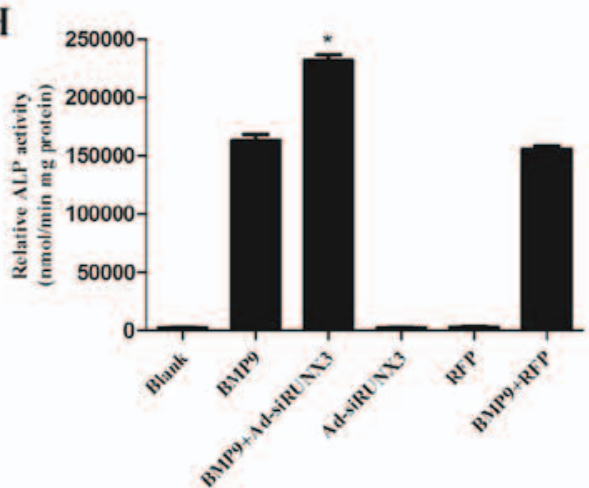

Figure 2. Effect of runt-related transcription factor 3 (RUNX3) on bone morphogenetic protein 9 (BMP9)-induced alkaline phosphatase (ALP) activity in mesenchymal stem cells (MSCs). (A) Adenovirus (Ad)-RUNX3 enhanced BMP9-induced ALP activity in C3H10T1/2 cells, asdetected by staining assay, magnification, x100. (B) Ad-RUNX3 enhanced BMP9-induced ALP activity in C3H10T1/2 cells, as detected by quantitive assay. (C) Adenovirus expressing small interfering RNA (siRNA) targeted against RUNX3 (Ad-siRUNX3) enhanced BMP9-induced ALP activity in C3H10T1/2 cells, as detected by staining assay. (D) Ad-siRUNX3 enhanced BMP9-induced ALP activity in C3H10T1/2 cells, as detected by quantitive assay. (E) Ad-RUNX3 enhanced BMP9-induced ALP activity in $\mathrm{C} 2 \mathrm{C} 12$ cells, as detected by staining assay. (F) Ad-RUNX3 enhanced BMP9-induced ALP activity in C2C12 cells, as detected by quantitive assay. (G) Ad-siRUNX3 enhanced BMP9-induced ALP activity in C2C12 cells, as detected by staining assay. (H) Ad-siRUNX3 enhanced BMP9-induced ALP activity in $\mathrm{C} 2 \mathrm{C} 12$ cells, as detected by quantitive assay. Magnification, $\mathrm{x} 100 .{ }^{*} \mathrm{P}<0.05$ vs. BMP9 group.

mineralization. We found that transfection with Ad-RUNX3 increased BMP9-induced matrix mineralization in C3H10T1/2 cells (Fig. 3A-a) and C2C12 cells (Fig. 3B-a). On the contrary however, transfection with Ad-siRUNX3 decreased BMP9induced matrix mineralization, as shown in the $\mathrm{C} 3 \mathrm{H} 10 \mathrm{~T} 1 / 2$ cells (Fig. 3A-b) and C2C12 cells (Fig. 3B-b). These results 
A
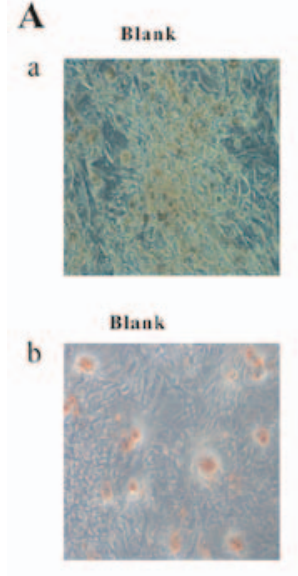

B

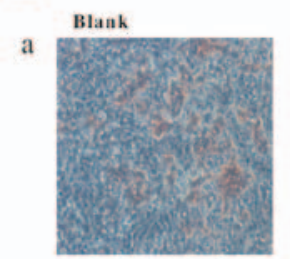

b

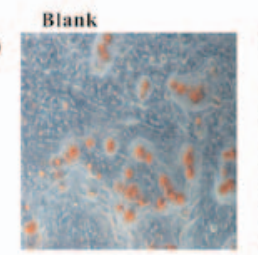

a
BMP9

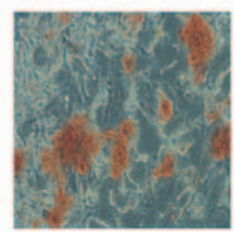

BMP9

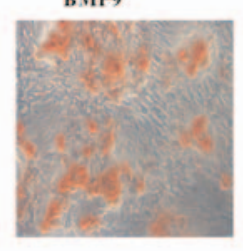

BMP9

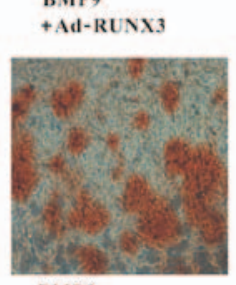

BMPe

+Ad-siRUNX3

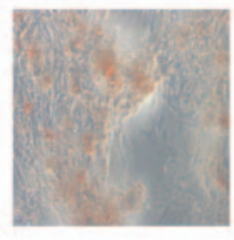

Ad-RUNX3

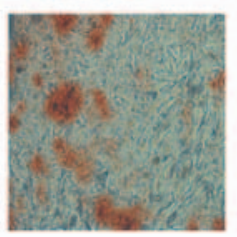

Ad-siRUNX3

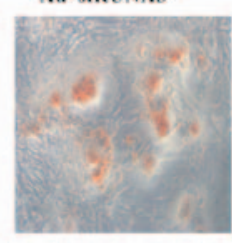

RFP

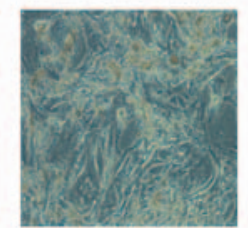

RFP

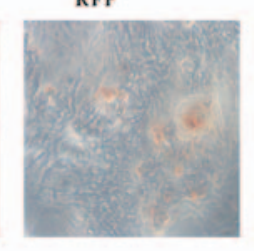

BMP9+ RFP

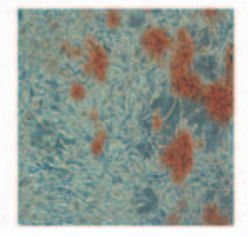

BMP9+RFP
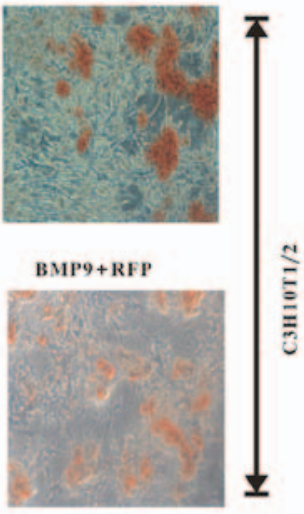

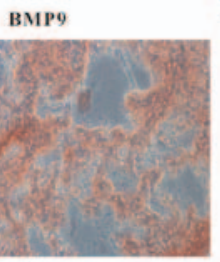

BMP9
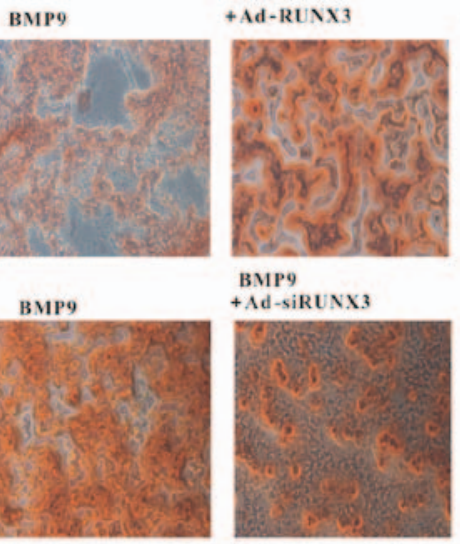

BMP9

+Ad-siRUNX3

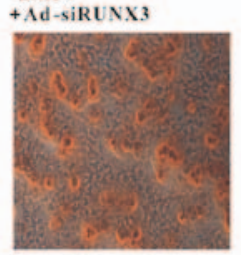

Ad-RUNX3
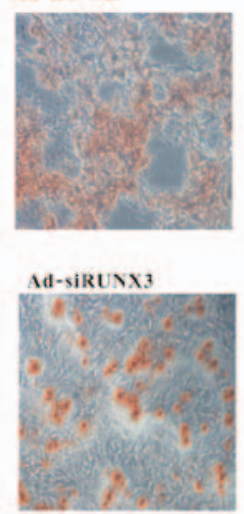

RFP

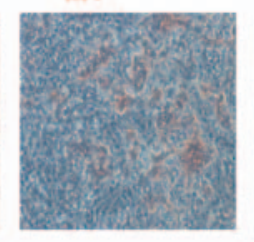

RFP

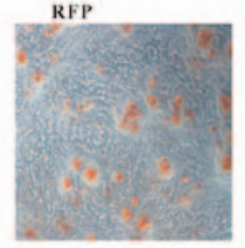

BMP9+RFP

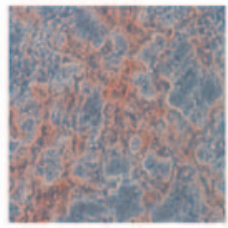

BMP9+RFP

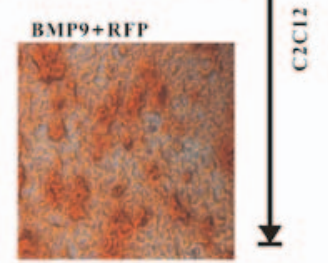

Figure 3. Effect of runt-related transcription factor 3 (RUNX3) on bone morphogenetic protein 9 (BMP9)-induced matrix mineralization of mesenchymal stem cells (MSCs). (A-a) Adenovirus (Ad)-RUNX3 enhanced BMP9-induced matrix mineralization in C3H10T1/2 cells, as detected by Alizarin Red S staining. (b) Adenovirus expressing small interfering RNA (siRNA) targeted against RUNX3 (Ad-siRUNX3) inhibited BMP9-induced matrix mineralization in C3H10T1/2 cells, as detected by Alizarin Red S staining. (B-a) Ad-RUNX3 enhanced BMP9-induced matrix mineralization in C2C12 cells, as detected by Alizarin Red S staining. (b) Ad-siRUNX3 inhibited BMP9-induced matrix mineralization in C2C12 cells, as detected by Alizarin Red S staining. Magnification, x100.

suggest that RUNX3 affects the BMP9-induced osteogenic differentiation of MSCs.

Effect of RUNX3 on the BMP9-induced expression of pivotal osteogenic transcription factors. It has previously been demonstrated that a number of pivotal osteogenic transcription factors, such as Id1, Id2, Id3, DLX5 and RUNX2 are involved in the BMP9-induced osteogenic differentiation of MSCs (39). Thus, in order to evaluate the effect of RUNX3 on the BMP9-induced expression of pivotal osteogenic transcription factors, we infected the C3H10T1/2 cells with Ad-RUNX3, Ad-siRUNX3 or Ad-RFP and then exposed the cells to BMP9-CM. The gene expression levels of Id1, Id2, Id3, DLX5 and RUNX2 were determined by RT-wPCR and the protein levels of DLX5 and RUNX2 were determined by western blot analysis. We found that the expression levels of Id3, DLX5 and RUNX2 were significantly increased by infection with Ad-RUNX3 and decreased by infection with Ad-siRUNX3 (Fig. 4C-E). However, infection with Ad-RUNX3 and Ad-siRUNX3 both increased the BMP9induced expression of Id1, Id2 (Fig. 4A and B). Subsequently, we further examined the protein expression levels of DLX5 and RUNX2 by western blot analysis, and found that Ad-RUNX3 increased the BMP9-induced expression of DLX5 and
RUNX2, while Ad-siRUNX3 decreased the expression of DLX5 and RUNX2 at the protein level (Fig. 4F). Collectively, these results suggest that RUNX3 is involved in regulating the BMP9-induced osteogenic differentiation of MSCs.

Effect of RUNX3 on BMP9-induced classical Smad-dependent signaling and Smad-independent MAPK signaling. We then sought to examine the possible mechanisms behind the effect of RUNX3 on the BMP9-induced osteogenic differentiation of MSCs. Previous studies have reported that the Smad-dependent signaling pathway and Smad-independent MAPK pathways are crucial in regulating BMP9 osteoinductive signaling (17-19). Thus, we wished to confirm whether RUNX3 can affect BMP9-activated Smad signaling and MAPK signaling in MSCs. We infected the C3H10T1/2 cells with Ad-RUNX3 or/and Ad-siRUNX3 (Fig. 5A). We found that Ad-RUNX3 increased the BMP9-induced phosphorylation of Smad1/5/8, whereas Ad-siRUNX3 decreased the BMP9-induced phosphorylation of Smad1/5/8 (Fig. 5A and B). We also found that BMP9 simultaneously stimulated the phosphorylation of p38 and ERK1/2 in the osteogenic differentiation process of MSCs (Fig. 5C and D), consistent with previous results (18). However, RUNX3 did not alter the BMP9-induced activation 

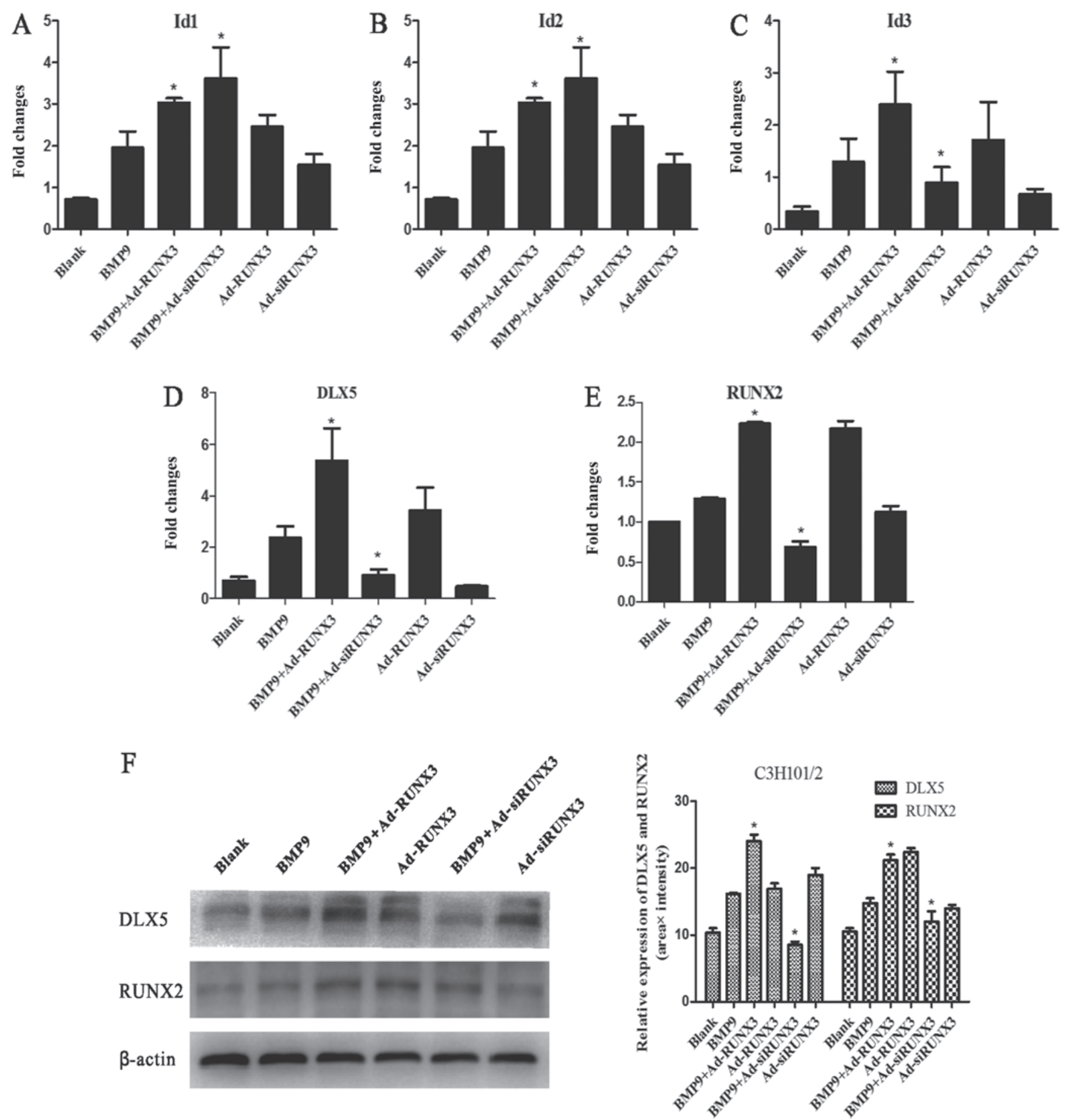

Figure 4. Effect of runt-related transcription factor 3 (RUNX3) on pivotal transcription factors during bone morphogenetic protein 9 (BMP9)-induced osteogenic differentiation of mesenchymal stem cells (MSCs). (A) Effect of RUNX3 on THE BMP9-induced gene expression level of Id1 in C3H10T1/2 cells measured by RT-qPCR. (B) Effect of RUNX3 on BMP9-induced gene expression level of Id2 in C3H10T1/2 cells measured by RT-qPCR. (C) Effect of RUNX3 on BMP9-induced gene expression level of Id3 in C3H10T1/2 cells measured by RT-qPCR. (D) Effect of RUNX3 on the BMP9-induced gene expression level of DLX5 measured by RT-qPCR. (E) Effect of RUNX3 on BMP9-induced gene expression level of RUNX2 measured by RT-qPCR. (F) Effect of RUNX3 on BMP9-induced protein level of DLX5 and RUNX2 measured by western blot analysis. Representative bands and a corresponding bar diagram are shown. " $\mathrm{P}<0.05$ vs. BMP9 group.

of p38 and ERK1/2 (Fig. 5C and D). These results indicate that RUNX3 regulates the BMP9-induced differentiation of MSCs via canonical Smad signaling.

\section{Discussion}

BMP9 [also known as growth differentiation factor-2 (GDF-2)], was originally isolated from fetal mouse liver and has been shown to stimulate hepatocyte proliferation (38). Other roles of BMP9 include regulating glucose and lipid metabolism in the liver (39), inducing the cholinergic phenotype of embryonic basal forebrain cholinergic neurons (40) and maintaining homeostasis of iron metabolism (41). Previously, BMP9 was identified as one of the most potent osteogenic BMPs both in vitro and in vivo (16), and yet it remains one of the least characterized BMPs. RUNX family members are similar in 
A
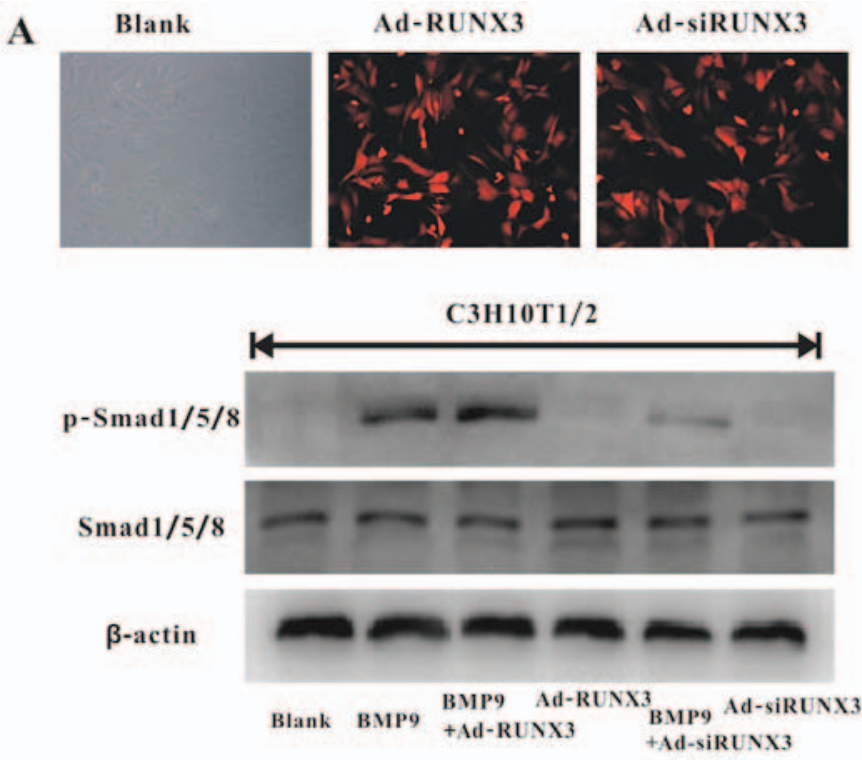

C

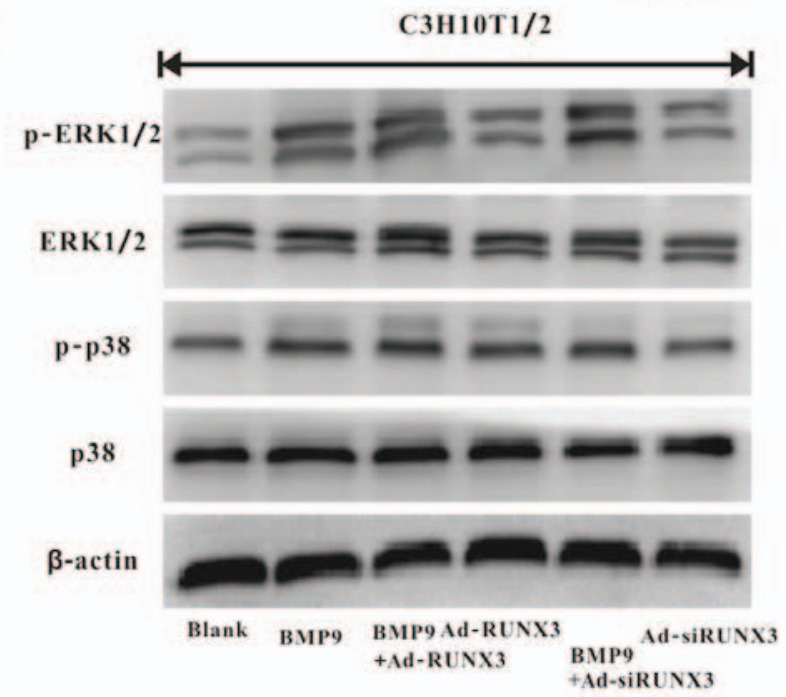

B

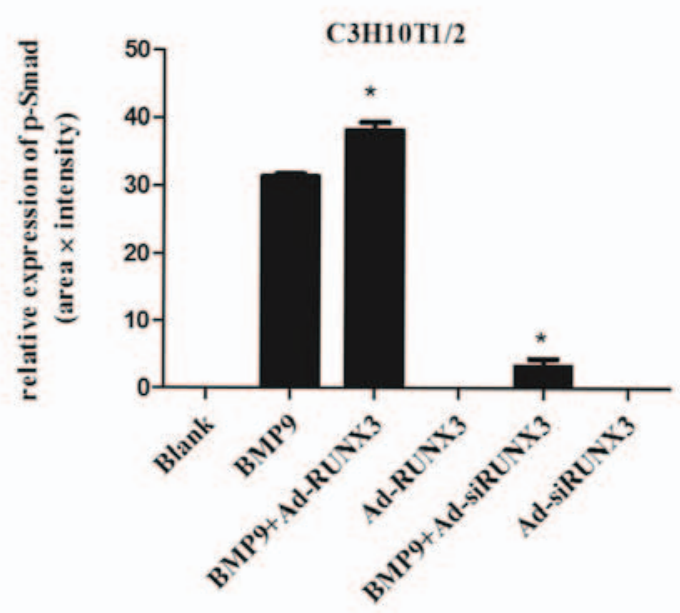

D

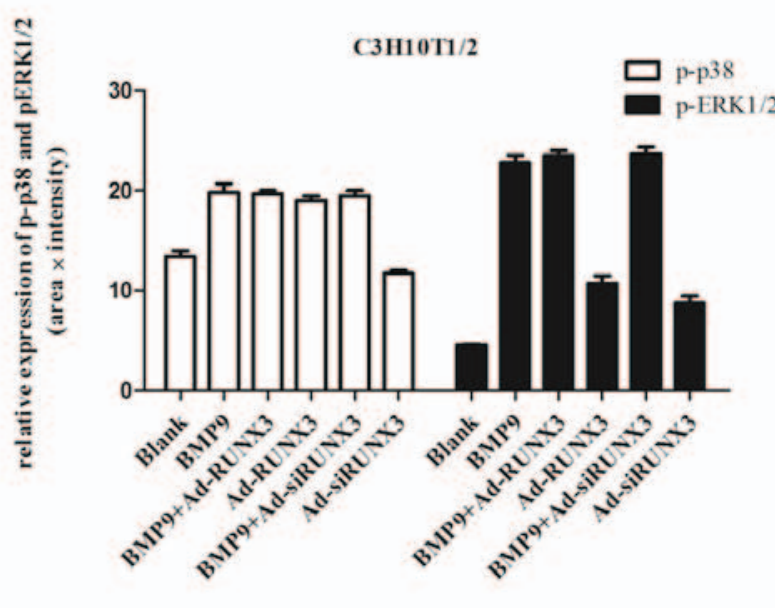

Figure 5. Effect of runt-related transcription factor 3 (RUNX3) on bone morphogenetic protein 9 (BMP9)-induced Smad-dependent and Smad-independent MAPK pathways. (A) Adenovirus (Ad)-RUNX3 and adenovirus expressing small interfering RNA (siRNA) targeted against RUNX3 (Ad-siRUNX3) were transfected into the C3H10T1/2 cells (MOI=5). Magnification, x100. (B) Effect of RUNX3 on BMP9-activitated Smad signaling. Representative bands and a corresponding bar diagram are shown. (C) Effect of RUNX3 on BMP9-induced p38 and ERK1/2. Representative bands and a corresponding bar diagram are shown in (D). "P<0.05 vs. BMP9 group.

mRNA and protein structure and are co-distributed in skeletal elements (33-35). It also has been reported that RUNX2 is essential for osteogenesis and is involved in BMP9-induced osteogenesis $(16,26,27,36)$. Although RUNX3 is an important regulator during chondrocyte differentiation and bone formation (42-44), the detailed role of RUNX3 in BMP9-induced osteogenesis is largely unknown. In the present study, we found RUNX3 regulated BMP9-induced osteogenesis via Smad signaling.

BMP9 was found to increase endogenous RUNX3 expression in MSCs in the present study. Notably, the overexpression of RUNX3 and the knockdown of RUNX3 both led to a potent promotion of ALP levels. RUNX1, RUNX2 and RUNX3 are all expressed in mesenchymal condensation (34). It has been previously demonstrated that after achieving the knockdown of one of the three RUNX members, the remaining RUNX proteins may compensate for the lack of the targeted one (32). Therefore, we speculate that this redundant function among the RUNX family may lead to the increased ALP activity following the knockdown of RUNX3. BMP9-induced late osteogenic marker matrix mineralization was enhanced by the overexpression of RUNX3, whereas it was inhibited by the knockdown of RUNX3. We noticed that the results of the ALP content and matrix mineralization were not consistent. We therefore hypothesized that this phenomenon may due to the following reasons: i) the sustained expression of RUNX3 and the knockdown of RUNX3 cannot reproduce the spatial and temporal expression pattern of RUNX3 during osteogenic differentiation; ii) the redundant function among the RUNX family may exert a differential influence on ALP and matrix mineralization.

Many pivotal transcription factors, such as Id, DLX5 and RUNX2 are involved in the BMP9-induced osteogenesis of MSCs (11). DLX5 and RUNX2 are also key transcription factors associated with osteogenesis $(26,27,45)$. In this study, however, we find that the transcriptional levels of Id1 and Id2 
were both upregulated by the overexpression and/or knockdown of RUNX3. We speculate that the redundant function among the RUNX family may have lead to this phenomenon. The results revealed that RUNX3 regulated important transcription factors involved in the BMP9-induced osteogenesis of MSCs.

We validated that Smad1/5/8, ERK1/2, p38 and JNKs are all involved in BMP9 osteoiductive signaling (17-19), implying that Smad-dependent and Smad-independent MAPK pathways are most important in regulating the BMP9-induced osteogenic differentiation of MSCs. In the present study, we found that the phosphorylation of Smad1/5/8 was increased by the overexpression of RUNX3 and was inhibited by the knockdown of RUNX3. This result suggests that RUNX3 may affect BMP9-induced Smad signaling. One possible explanation of the RUNX3 effect on Smad signaling is that RUNX3 may facilitate Smad1/5/8 to bind to BMP type I receptor which can directly phosphorylate Smad1/5/8. Additionally, nuclear export factor RanBP3 can regulate the phosphorylation of Smad1/5/8 by controlling the nucleocytoplasmic shuttling of Smad1, Smad5 and Smad8 (Smad1/5/8) (46,47). Nuclear phosphatases, such as PPM1A can also affect the phosphorylation of Smad1/5/8 by dephosphorylating Smad1/5/8 $(48,49)$. Therefore another explanation of the RUNX3 effect on Smad signaling is that RUNX3 may interact with RanBP3 and/or PPM1A to affect Smad1/5/8 phosphorylation. BMP9 can simultaneously stimulate the phosphorylation/ activation of p38 and ERK1/2 (18). However, RUNX3 did not affect the BMP9-induced phosphorylation of p38 and ERK1/2. Taken together, our results strongly indicate that RUNX3 is likely to play regulatory roles in the BMP9-induced osteogenic differentiation of MSCs mainly by affecting the Smad signaling cascade.

Previous studies have demonstrated that RUNX3 is an important regulator of chondrocyte differentiation (30-32,50,51). The loss of osteoblastic RUNX3 produces severe congenital osteopenia (33). To date, little is known about RUNX3 during osteogenesis. In this study, we explored the detailed roles of RUNX3 during the BMP9-induced osteogenic differentiation of MSCs. We found that RUNX3 played an important role during BMP9-induced osteogenesis. These finding may contribute not only to enrich our understanding of the osteoinductive activity of BMP9, but may also provide a solid basis for the use of BMP9 in bone tissue engineering. Future studies are warranted to identity the direct target gene of RUNX3 during BMP9-induced osteogenesis and explore the precise mechanisms responsible for the effects of RUNX3 on the phosphorylation of Smad1/5/8.

\section{Acknowledgements}

This study was supported by grants from the National Natural Science Foundation of China (no. 81272006) and the Natural Science Foundation Project of Chongqing Science and Technology Commission (no. cstc2013jcyjA10061)

\section{References}

1. Deschaseaux F, Pontikoglou C and Sensébé L: Bone regeneration: The stem/progenitor cells point of view. J Cell Mol Med 14: 103-115, 2010.

2. Shenaq DS, Rastegar F, Petkovic D, Zhang BQ, He BC, Chen L, Zuo GW, Luo Q, Shi Q, Wagner ER, et al: Mesenchymal progenitor cells and their orthopedic applications: Forging a path towards clinical trials. Stem Cells Int 2010: 519028, 2010.
3. Thakker R and Yang P: Mesenchymal stem cell therapy for cardiac repair. Curr Treat Options Cardiovasc Med 16: 323, 2014.

4. Myers TJ, Granero-Molto F, Longobardi L, Li T, Yan Y and Spagnoli A: Mesenchymal stem cells at the intersection of cell and gene therapy. Expert Opin Biol Ther 10: 1663-1679, 2010.

5. García-Gómez I, Elvira G, Zapata AG, Lamana ML, Ramírez M, Castro JG, Arranz MG, Vicente A, Bueren J and García-Olmo D: Mesenchymal stem cells: Biological properties and clinical applications. Expert Opin Biol Ther 10: 1453-1468, 2010.

6. Deng ZL, Sharff KA, Tang N, Song WX, Luo J, Luo X, Chen J, Bennett E, Reid R, Manning D, et al: Regulation of osteogenic differentiation during skeletal development. Front Biosci 13: 2001-2021, 2008

7. Arthur A, Zannettino A and Gronthos S: The therapeutic applications of multipotential mesenchymal/stromal stem cells in skeletal tissue repair. J Cell Physiol 218: 237-245, 2009.

8. Stappenbeck TS and Miyoshi H: The role of stromal stem cells in tissue regeneration and wound repair. Science 324: 1666-1669, 2009.

9. Botchkarev VA: Bone morphogenetic proteins and their antagonists in skin and hair follicle biologyn. J Invest Dermatol 120: 37-47, 2003.

10. Zhao GQ: Consequences of knocking out BMP signaling in the mouse. Genesis 35: 43-56, 2003.

11. Luu HH, Song WX, Luo X, Manning D, Luo J, Deng ZL, Sharff KA, Montag AG, Haydon RC and He TC: Distinct roles of bone morphogenetic proteins in osteogenic differentiation of mesenchymal stem cells. J Orthop Res 25: 665-677, 2007.

12. Varady P, Li JZ, Alden TD, Kallmes DF, Williams MB and Helm GA: CT and radionuclide study of BMP-2 gene therapyinduced bone formation. Acad Radiol 9: 632-637, 2002.

13. Krebsbach PH, Gu K, Franceschi RT and Rutherford RB: Gene therapy-directed osteogenesis: BMP-7-transduced human fibroblasts form bone in vivo. Hum Gene Ther 11: 1201-1210, 2000.

14. Boraiah S, Paul O, Hawkes D, Wickham M and Lorich DG: Complications of recombinant human BMP-2 for treating complex tibial plateau fractures: A preliminary report. Clin Orthop Relat Res 467: 3257-3262, 2009.

15. Rutherford RB, Nussenbaum B and Krebsbach PH: Bone morphogenetic protein 7 ex vivo gene therapy. Drug News Perspect 16: 5-10, 2003.

16. Kang Q, Sun MH, Cheng H, Peng Y, Montag AG, Deyrup AT, Jiang W, Luu HH, Luo J, Szatkowski JP, et al: Characterization of the distinct orthotopic bone-forming activity of 14 BMPs using recombinant adenovirus-mediated gene delivery. Gene Ther 11: 1312-1320, 2004

17. Zhao YF, Xu J, Wang WJ, Wang J, He JW, Li L, Dong Q, Xiao Y, Duan XL, Yang X, et al: Activation of JNKs is essential for BMP9-induced osteogenic differentiation of mesenchymal stem cells. BMB Rep 46: 422-427, 2013.

18. Zhao Y, Song T, Wang W, Wang J, He J, Wu N, Tang M, He B and Luo J: P38 and ERK1/2 MAPKs act in opposition to regulate BMP9-induced osteogenic differentiation of mesenchymal progenitor cells. PLoS One 7: e43383, 2012.

19. Xu DJ, Zhao YZ, Wang J, He JW, Weng YG and Luo JY: Smads, p38 and ERK1/2 are involved in BMP9-induced osteogenic differentiation of C3H10T1/2 mesenchymal stem cells. BMB Rep 45: 247-252, 2012.

20. Luo J, Tang M, Huang J, He BC, Gao JL, Chen L, Zuo GW, Zhang W, Luo Q, Shi Q, et al: TGFbeta/BMP type I receptors ALK1 and ALK2 are essential for BMP9-induced osteogenic signaling in mesenchymal stem cells. J Biol Chem 285: 29588-29598, 2010.

21. Zhang W, Zhang L, Zhou Y, Ji X, Liu J, Liu D, Yin P, Peng Y, Hao M, Zhang L, et al: Synergistic effects of BMP9 and miR-548d-5p on promoting osteogenic differentiation of mesenchymal stem cells. Biomed Res Int 2015: 309747, 2015.

22. Zhang R, Weng Y, Li B, Jiang Y, Yan S, He F, Chen X, Deng F, Wang J and Shi Q: BMP9-induced osteogenic differentiation is partially inhibited by miR-30a in the mesenchymal stem cell line C3H10T1/2. J Mol Histol 46: 399-407, 2015.

23. Song Q, Zhong L, Chen C, Tang Z, Liu H, Zhou Y, Tang M,Zhou L, Zuo G, Luo J, et al: miR-21 synergizes with BMP9 in osteogenic differentiation by activating the BMP9/Smad signaling pathway in murine multilineage cells. Int J Mol Med 36: 1497-1506, 2015.

24. Choi JY, Pratap J, Javed A, Zaidi SK, Xing L, Balint E, Dalamangas S, Boyce B, van Wijnen AJ, Lian JB, et al: Subnuclear targeting of Runx/Cbfa/AML factors is essential for tissue-specific differentiation during embryonic development. Proc Natl Acad Sci USA 98: 8650-8655, 2001. 
25. Wang Q, Stacy T, Binder M, Marin-Padilla M, Sharpe AH and Speck NA: Disruption of the Cbfa2 gene causes necrosis and hemorrhaging in the central nervous system and blocks definitive hematopoiesis. Proc Natl Acad Sci USA 93: 3444-3449, 1996.

26. Otto F, Thornell AP, Crompton T, Denzel A, Gilmour KC, Rosewell IR, Stamp GW, Beddington RS, Mundlos S, Olsen BR, et al: Cbfa1, a candidate gene for cleidocranial dysplasia syndrome, is essential for osteoblast differentiation and bone development. Cell 89: 765-771, 1997.

27. Komori T, Yagi H, Nomura S, Yamaguchi A, Sasaki K, Deguchi K, Shimizu Y, Bronson RT, Gao YH, Inada M, et al: Targeted disruption of Cbfal results in a complete lack of bone formation owing to maturational arrest of osteoblasts. Cell 89: 755-764, 1997.

28. Levanon D, Bettoun D, Harris-Cerruti C, Woolf E, Negreanu V, Eilam R, Bernstein Y, Goldenberg D, Xiao C, Fliegauf M, et al: The Runx3 transcription factor regulates development and survival of TrkC dorsal root ganglia neurons. EMBO J 21: 3454-3463, 2002.

29. Brenner O, Levanon D, Negreanu V, Golubkov O, Fainaru O, Woolf $\mathrm{E}$ and Groner Y: Loss of Runx 3 function in leukocytes is associated with spontaneously developed colitis and gastric mucosal hyperplasia. Proc Natl Acad Sci USA 101: 16016-16021, 2004.

30. Soung Y, Dong Y, Wang Y, Zuscik MJ, Schwarz EM, O'Keefe RJ and Drissi H: Runx3/AML2/Cbfa3 regulates early and late chondrocyte differentiation. J Bone Miner Res 22: 1260-1270, 2007.

31. Yoshida CA and Komori T: Role of Runx proteins in chondrogenesis. Crit Rev Eukaryot Gene Expr 15: 243-254, 2005.

32. Stricker S, Fundele R, Vortkamp A and Mundlos S: Role of Runx genes in chondrocyte differentiation. Dev Biol 245: 95-108, 2002.

33. Bauer O, Sharir A, Kimura A, Hantisteanu S, Takeda S and Groner Y: Loss of osteoblast Runx3 produces severe congenital osteopenia. Mol Cell Biol 35: 1097-1109, 2015.

34. Levanon D and Groner Y: Structure and regulated expression of mammalian RUNX genes. Oncogene 23: 4211-4219, 2004.

35. Levanon D, Brenner O, Negreanu V, Bettoun D, Woolf E, Eilam R, Lotem J, Gat U, Otto F, Speck N, et al: Spatial and temporal expression pattern of Runx3 (Aml2) and Runx1 (Aml1) indicates non-redundant functions during mouse embryogenesis. Mech Dev 109: 413-417, 2001.

36. Sharff KA, Song WX, Luo X, Tang N, Luo J, Chen J, Bi Y, He BC, Huang J, Li X, et al: Heyl basic helix-loop-helix protein plays an important role in mediating BMP9-induced osteogenic differentiation of mesenchymal progenitor cells. J Biol Chem 284: 649-659, 2009

37. He TC, Zhou S, da Costa LT, Yu J, Kinzler KW and Vogelstein B: A simplified system for generating recombinant adenoviruses. Proc Natl Acad Sci USA 95: 2509-2514, 1998.

38. Song JJ, Celeste AJ, Kong FM, Jirtle RL, Rosen V and Thies RS: Bone morphogenetic protein-9 binds to liver cells and stimulates proliferation. Endocrinology 136: 4293-4297, 1995.
39. Chen C, Grzegorzewski KJ, Barash S, Zhao Q, Schneider H, Wang Q, Singh M, Pukac L, Bell AC, Duan R, et al: An integrated functional genomics screening program reveals a role for BMP-9 in glucose homeostasis. Nat Biotechnol 21: 294-301, 2003.

40. López-Coviella I, Berse B, Krauss R, Thies RS and Blusztajn JK: Induction and maintenance of the neuronal cholinergic phenotype in the central nervous system by BMP-9. Science 289: 313-316, 2000.

41. Truksa J, Peng H, Lee P and Beutler E: Bone morphogenetic proteins 2,4 , and 9 stimulate murine hepcidin 1 expression independently of Hfe, transferrin receptor 2 (Tfr2), and IL-6. Proc Natl Acad Sci USA 103: 10289-10293, 2006.

42. Smith N, Dong Y, Lian JB, Pratap J, Kingsley PD, van Wijnen AJ, Stein JL, Schwarz EM, O'Keefe RJ, Stein GS, et al: Overlapping expression of Runx1(Cbfa2) and Runx2 (Cbfa1) transcription factors supports cooperative induction of skeletal development. J Cell Physiol 203: 133-143, 2005.

43. Yoshida CA, Yamamoto H, Fujita T, Furuichi T, Ito K, Inoue K, Yamana K, Zanma A, Takada K, Ito Y, et al: Runx2 and Runx3 are essential for chondrocyte maturation, and Runx 2 regulates limb growth through induction of Indian hedgehog. Genes Dev 18: 952-963, 2004.

44. Kim EJ, Cho SW, Shin JO, Lee MJ, Kim KS and Jung HS: Ihh and Runx2/Runx3 signaling interact to coordinate early chondrogenesis: A mouse model. PLoS One 8: e55296, 2013.

45. Ferrari D, Harrington A, Dealy CN and Kosher RA: Dlx-5 in limb initiation in the chick embryo. Dev Dyn 216: 10-15, 1999.

46. Chen F, Lin X, Xu P, Zhang Z, Chen Y, Wang C, Han J, Zhao B, Xiao $\mathrm{M}$ and Feng XH: Nuclear export of smads by ranBP3L regulates bone morphogenetic protein signaling and mesenchymal stem cell differentiation. Mol Cell Biol 35: 1700-1711, 2015.

47. Dai F, Lin X, Chang C and Feng XH: Nuclear export of Smad2 and Smad3 by RanBP3 facilitates termination of TGF-beta signaling. Dev Cell 16: 345-357, 2009.

48. Lin X, Duan X, Liang YY, Su Y, Wrighton KH, Long J, Hu M, Davis CM, Wang J, Brunicardi FC, et al: PPM1A functions as a Smad phosphatase to terminate TGFbeta signaling. Cell 125: 915-928, 2006

49. Dai F, Shen T, Li Z, Lin X and Feng XH: PPM1A dephosphorylates RanBP3 to enable efficient nuclear export of Smad2 and Smad3. EMBO Rep 12: 1175-1181, 2011.

50. Wigner NA, Soung Y, Einhorn TA, Drissi H and Gerstenfeld LC: Functional role of Runx3 in the regulation of aggrecan expression during cartilage development. J Cell Physiol 228: 2232-2242, 2013.

51. Dalcq J, Pasque V, Ghaye A, Larbuisson A, Motte P, Martial JA and Muller M: RUNX3, EGR1 and SOX9B form a regulatory cascade required to modulate BMP-signaling during cranial cartilage development in zebrafish. PLoS One 7: e50140, 2012. 